\title{
Economic Analysis of Integrated Weed Management in Brinjal Crop (Solanum melongena L.)
}

\author{
Sumeet Singh $^{1 *}$, Kulbir Singh ${ }^{1}$, D.S. Khurana ${ }^{2}$ and V. Sardana ${ }^{2}$ \\ ${ }^{1}$ Department of Vegetable Science, PAU, Ludhiana-141004 Punjab, India \\ ${ }^{2}$ Department of Plant Breeding and Genetics, PAU, Ludhiana-141004, Punjab, India \\ *Corresponding author
}

\begin{tabular}{l} 
K e y w o r d s \\
Brinjal, Mulch, \\
Herbicide, \\
Integrated weed \\
management, \\
Pendimethalin, \\
Oxyflourfen and \\
cost benefit ratio. \\
\hline Article Info \\
$\begin{array}{l}\text { Accepted: } \\
\text { 10 October } 2017 \\
\text { Available Online: } \\
\text { 10 December } 2017\end{array}$ \\
\hline
\end{tabular}

\section{Keywords}

Brinjal, Mulch, Herbicide, Integrated weed agement, cost benefit ratio.

Accepted: Available Online: December 2017

\section{Introduction}

Being one of the most important vegetable crops, brinjal has been under cultivation since ancient times in many tropical countries and widely cultivated in the central, southern and south-east Asian region. It is also popular in other countries of the world like France, Italy and United States (Singh and Kalda 2001).India is regarded as the centre of origin of brinjal (Vavilov, 1931; Bhaduri, 1951) where it has been cultivated in India for the last 4,000 years, although it is often thought of as a Mediterranean or mid-Eastern vegetable. After potato, it is the second highest consumed vegetable in India along with tomato and onion and is considered as poor's man vegetable. Brinjal is quite high in nutritive value and can be well compared with tomato and is consumed as a cooked vegetable in various ways. Although, considerable research has been done in India on various aspects of brinjal cultivation yet information on its weed management is inadequate. It has been estimated that losses in yield due to weeds alone vary from 10 to 
$70 \%$ depending upon the extent of weed infestation (Mani et al., 1968). Wider spacing, slow initial growth, long duration, frequent irrigations and liberal use of farmyard manure and chemical fertilizers encourage fast growth and development of weeds in brinjal even frequent irrigations encourage germination of weed seeds in many flushes (Walia, 2009). Growth of weeds in early stage is more critical than weeds at later stages (Kumar and Pratap, 1993) and poses more threat economically. So, there is need to integrate management practices with proper weed control method which are economically more viable being a prime need and much essential for obtaining potential yield. So, present investigation was designed using chemical herbicides, hand hoeing, plastic mulch as well as solarization for as integrated approach.

\section{Materials and Methods}

The experiment was conducted at Vegetable Research Farm, Department of Vegetable science, Punjab Agricultural University, Ludhiana. Ludhiana is characterized by hot summer and cold winter with semi-arid and sub-tropical climate, which represents a typical monsoon conditions prevailing in central districts of Punjab.

The mean maximum and minimum temperature show considerable fluctuations during summer, while minimum temperature falls below freezing point accompanied by frosty spells during winter. The average rainfall is about $500-700 \mathrm{~mm}$, most of which is normally received from July-September. Randomized Complete Block Design with three replications having fifteen different treatments comprising of hand weeding, mulching, pre-transplanting treatments with trifluralin $(0.240 \mathrm{~kg} / \mathrm{ha}$ and $0.300 \mathrm{~kg} / \mathrm{ha})$, pendimethalin $(0.56 \mathrm{~kg} / \mathrm{ha}$ and $0.75 \mathrm{~kg} / \mathrm{ha})$, oxyfluorfen $(0.15 \mathrm{~kg} / \mathrm{ha}$ and $0.20 \mathrm{~kg} / \mathrm{ha})$ and post-transplanting treatment with paraquat
$(1.0 \mathrm{~kg} / \mathrm{ha})$ and un-weeded check were used. Blanket application of herbicides were sprayed two days before transplanting in 500 liters of water per ha with knapsack sprayer using cut nozzle. Brinjal hybrid 'BH-6747' was used as a planting material and observations on various growth and yield parameters of crop viz., plant height, number of branches per plant, number and weight of fruits per plant, fruit yield as well as observations on weeds such as weed count, dry matter of weeds and weed control efficiency as influenced by various treatments were recorded and economics was calculated. One month old seedlings were planted at a spacing of $67 \mathrm{x} 45 \mathrm{~cm}$ in field. The recommended package of practices was followed for raising the crop (Anonymous 2010).

\section{Results and Discussion}

During kharif season brinjal field is highly infested with weeds due to wider spacing, on set of monsoon and slow growth of crop cause a challenge for managing weeds. Many weed species were observed in field but major weeds were Cynodon dactylon, Eleusine indica, Cyperus rotundus, Phyllanthus niruri, Trianthema portulacastrum, Portulaca oleracea, Parthenium hysterophorus, Euphorbia hirta, Amaranthus viridi and Convolvulus arvensis. These weeds highly effected the growth of plants but treatments showed significant effect on weed number, dry matter accumulation, and weed control efficiency as well as on weed index. Weeds in nursery were significantly controlled by Trifluralin (pre-plant incorporation) @ 0.240 $\mathrm{kg}$ a.i /ha although maximum germination was recorded in solarozation but Trifluralin (pre-plant incorporation) @ $0.240 \mathrm{~kg}$ a.i /ha was concluded as a best remedy for nursery (Table 1). Germination was severly affected by oxyflourfen @ $0.175 \mathrm{~kg}$ a.i /ha and recorded lowest germination. 
Int.J.Curr.Microbiol.App.Sci (2017) 6(12): 1245-1251

Table.1 Effect of weed control treatments on weed count, fresh weight, dry weight and germination (\%) on brinjal nursery

\begin{tabular}{|c|c|c|c|c|c|}
\hline & Treatment & $\begin{array}{l}\text { Weed count } \\
\qquad\left(\mathrm{m}^{2}\right)\end{array}$ & $\begin{array}{l}\text { Fresh weight } \\
\qquad\left(\mathrm{g} / \mathrm{m}^{2}\right)\end{array}$ & $\begin{array}{l}\text { Dry weight } \\
\qquad\left(\mathrm{g} / \mathrm{m}^{2}\right)\end{array}$ & Germination $(\%)$ \\
\hline $\mathrm{T}_{1}$ & Solarization (for 30 days) & 34.6 & 366.6 & 98.3 & 78.40 \\
\hline $\mathrm{T}_{2}$ & $\begin{array}{l}\text { Pendimethalin (pre-emergence) @ } \\
0.225 \mathrm{~kg} \text { a.i. /ha }\end{array}$ & 21.0 & 295.0 & 88.3 & 75.00 \\
\hline $\mathrm{T}_{3}$ & $\begin{array}{l}\text { Pendimethalin (pre-emergence) @ } \\
0.300 \mathrm{~kg} \text { a.i /ha }\end{array}$ & 19.0 & 276.6 & 81.6 & 65.33 \\
\hline $\mathrm{T}_{4}$ & $\begin{array}{l}\text { Oxyfluorfen (pre-emergence) @ } 0.120 \\
\mathrm{~kg} \mathrm{a.i} \mathrm{/ha}\end{array}$ & 18.6 & 166.6 & 45.0 & 54.33 \\
\hline $\mathrm{T}_{5}$ & $\begin{array}{l}\text { Oxyfluorfen (pre-emergence) @ } 0.175 \\
\mathrm{~kg} \mathrm{a.i} \mathrm{/ha}\end{array}$ & 11.3 & 160.0 & 43.3 & 31.00 \\
\hline $\mathrm{T}_{6}$ & $\begin{array}{l}\text { Trifluralin (pre-plant incorporation) @ } \\
0.240 \mathrm{~kg} \mathrm{a.i} \mathrm{/ha}\end{array}$ & 20.0 & 233.3 & 56.6 & 72.00 \\
\hline $\mathrm{T}_{7}$ & $\begin{array}{l}\text { Trifluralin (pre-plant incorporation) @ } \\
0.300 \mathrm{~kg} \text { a.i /ha }\end{array}$ & 17.0 & 223.3 & 55.0 & 68.40 \\
\hline $\mathrm{T}_{8}$ & Un-weeded & 82.0 & 761.6 & 266.6 & 32.67 \\
\hline \multirow[t]{2}{*}{$\mathrm{T}_{9}$} & Weed free (15 days interval) & 0.1 & 0.1 & 0.1 & 80.33 \\
\hline & $\mathrm{C} \mathrm{D}(\mathrm{P}=0.05)$ & 5.3 & 50.2 & 14.8 & 5.32 \\
\hline
\end{tabular}


Table.2 Effect of weed control treatments on weed count, weed dry weight, weed control efficiency at different intervals and weed index

\begin{tabular}{|c|c|c|c|c|c|c|c|c|c|c|}
\hline \multirow[t]{2}{*}{ Treatment } & \multicolumn{3}{|c|}{$\begin{array}{l}\text { Weed count } \\
\left(\text { number } / \mathrm{m}^{2}\right)\end{array}$} & \multicolumn{3}{|c|}{ Dry weight $\left(\mathrm{g} / \mathrm{m}^{2}\right)$} & \multicolumn{3}{|c|}{$\begin{array}{c}\text { Weed control efficiency } \\
(\%)\end{array}$} & \multirow{2}{*}{$\begin{array}{l}\text { Weed } \\
\text { Index } \\
(\%)\end{array}$} \\
\hline & $\begin{array}{c}30 \\
\text { DAT }\end{array}$ & $\begin{array}{c}60 \\
\text { DAT }\end{array}$ & $\begin{array}{c}90 \\
\text { DAT }\end{array}$ & $\begin{array}{c}30 \\
\text { DAT }\end{array}$ & $\begin{array}{c}60 \\
\text { DAT }\end{array}$ & $\begin{array}{c}90 \\
\text { DAT }\end{array}$ & $\begin{array}{c}30 \\
\text { DAT }\end{array}$ & $\begin{array}{c}60 \\
\text { DAT }\end{array}$ & $\begin{array}{c}90 \\
\text { DAT }\end{array}$ & \\
\hline Pendimethalin (pre tranplant) @ 0.56 kg/ha & 38.0 & 217.0 & 329.0 & 81.6 & 116.6 & 155.0 & 81.46 & 31.11 & 19.95 & 13.66 \\
\hline Pendimethalin (pre tranplant) @ 0.75 kg/ha & 35.0 & 211.7 & 347.3 & 70.0 & 111.6 & 166.6 & 82.93 & 32.80 & 15.49 & 13.44 \\
\hline $\begin{array}{l}\text { Pendimethalin (pre tranplant) @ } 0.56 \mathrm{~kg} / \mathrm{ha}+ \\
\text { hoeing (45 DAT) }\end{array}$ & 40.7 & 91.0 & 209.7 & 71.6 & 55.3 & 81.6 & 80.16 & 71.11 & 48.99 & 4.85 \\
\hline $\begin{array}{l}\text { Pendimethalin (pre tranplant) @ } 0.56 \mathrm{~kg} / \mathrm{ha}+ \\
\text { paraquat } 1.0 \mathrm{~kg} / \mathrm{ha} \text { (directed spray at } 45 \mathrm{DAT})\end{array}$ & 33.3 & 161.0 & 240.0 & 76.6 & 65.0 & 125.0 & 83.74 & 48.89 & 41.61 & 7.07 \\
\hline Oxyfluorfen (pre tranplant) @ $0.15 \mathrm{~kg} / \mathrm{ha}$ & 39.3 & 198.0 & 313.7 & 71.6 & 105.0 & 161.6 & 80.81 & 37.14 & 23.68 & 15.84 \\
\hline Oxyfluorfen (pre tranplant) @ $0.20 \mathrm{~kg} / \mathrm{ha}$ & 30.0 & 113.0 & 308.7 & 65.0 & 103.3 & 160.0 & 85.37 & 64.13 & 24.90 & 14.19 \\
\hline $\begin{array}{l}\text { Oxyfluorfen (pre tranplant) @ } 0.15 \mathrm{~kg} / \mathrm{ha}+ \\
\text { hoeing (45 DAT) }\end{array}$ & 33.7 & 86.3 & 190.3 & 40.0 & 71.3 & 68.3 & 83.58 & 72.59 & 53.69 & 3.48 \\
\hline $\begin{array}{l}\text { Oxyfluorfen (pre tranplant) @ } 0.15 \mathrm{~kg} / \mathrm{ha}+ \\
\text { paraquat } 1.0 \mathrm{~kg} / \mathrm{ha} \text { (directed spray at } 45 \mathrm{DAT})\end{array}$ & 33.3 & 150.3 & 203.0 & 72.0 & 65.0 & 123.3 & 83.74 & 52.28 & 50.61 & 14.98 \\
\hline Trifluralin (pre tranplant) @60 kg/ha & 43.3 & 207.6 & 342.0 & 88.3 & 110.0 & 143.3 & 78.86 & 34.07 & 16.79 & 19.12 \\
\hline Trifluralin (pre tranplant) @ $0.90 \mathrm{~kg} / \mathrm{ha}$ & 40.0 & 191.6 & 324.0 & 75.0 & 91.6 & 108.3 & 85.49 & 39.15 & 21.17 & 16.29 \\
\hline $\begin{array}{l}\text { Trifluralin (pre tranplant) @ } 0.60 \mathrm{~kg} / \mathrm{ha}+ \\
\text { hoeing (45 DAT) }\end{array}$ & 41.3 & 112.3 & 206.3 & 83.6 & 62.0 & 95.0 & 89.84 & 64.34 & 49.80 & 6.83 \\
\hline $\begin{array}{l}\text { Trifluralin (pre tranplant) @ } 0.60 \\
\mathrm{~kg} / \mathrm{ha+paraquat} 1.0 \mathrm{~kg} / \mathrm{ha} \text { (directed spray at } 45 \\
\text { DAT) }\end{array}$ & 40.7 & 161.0 & 276.0 & 85.0 & 63.6 & 105.0 & 80.16 & 48.89 & 32.85 & 5.85 \\
\hline Black mulch & 5.0 & 10.3 & 14.0 & 10.0 & 25.0 & 20.0 & 97.56 & 96.72 & 96.59 & -0.73 \\
\hline Weed free & 0.0 & 0.0 & 0.0 & 0.0 & 0.0 & 0.00 & 100.00 & $\begin{array}{c}100.0 \\
0\end{array}$ & 100.00 & 0.00 \\
\hline Un-weeded & 205.0 & 315.0 & 411.0 & 218.3 & 228.6 & 253.3 & 0.00 & 0.00 & 0.00 & 32.71 \\
\hline $\mathrm{CD}(\mathrm{P}=0.05)$ & 6.8 & 8.6 & 8.6 & 8.6 & 24.4 & 25.5 & - & - & - & - \\
\hline
\end{tabular}


Table.3 Effect of weed control treatments on growth, yield attributes, fruit yield of brinjal and cost benefit ratio

\begin{tabular}{|c|c|c|c|c|c|c|c|c|c|c|}
\hline \multirow[b]{2}{*}{ Treatment } & \multicolumn{3}{|c|}{ Plant height $(\mathrm{cm})$} & \multirow{2}{*}{$\begin{array}{l}\text { Numb } \\
\text { er of } \\
\text { branch } \\
\text { es }\end{array}$} & \multirow{2}{*}{$\begin{array}{l}\text { Average } \\
\text { fruit } \\
\text { weight } \\
\text { (g) }\end{array}$} & \multirow[b]{2}{*}{$\begin{array}{c}\text { Fruits/ } \\
\text { plant }\end{array}$} & \multirow{2}{*}{$\begin{array}{l}\text { Cost of } \\
\text { treatment } \\
\quad(\mathrm{RS})\end{array}$} & \multirow{2}{*}{$\begin{array}{l}\text { Gross } \\
\text { returns } \\
\text { (Rs.) }\end{array}$} & \multirow{2}{*}{$\begin{array}{l}\text { Total } \\
\text { yield } \\
(\mathrm{q} / \mathrm{ha})\end{array}$} & \multirow{2}{*}{$\begin{array}{l}\mathrm{C}: \mathrm{B} \\
\text { ratio }\end{array}$} \\
\hline & $\begin{array}{c}30 \\
\text { DAT }\end{array}$ & $\begin{array}{c}60 \\
\text { DAT }\end{array}$ & $\begin{array}{c}90 \\
\text { DAT }\end{array}$ & & & & & & & \\
\hline Pendimethalin (pre tranplant) @ 0.56 kg/ha & 25.3 & 42.2 & 62.9 & 20.3 & 47.5 & 35.3 & 42902.50 & 218805.00 & 437.6 & $1: 5.1$ \\
\hline Pendimethalin (pre tranplant) @ $0.75 \mathrm{~kg} / \mathrm{ha}$ & 24.6 & 40.2 & 60.2 & 21.6 & 48.9 & 36.2 & 43217.50 & 219355.00 & 438.7 & $1: 5.0$ \\
\hline $\begin{array}{l}\text { Pendimethalin (pre tranplant) @ } 0.56 \mathrm{~kg} / \mathrm{ha} \\
\text { + hoeing ( } 45 \mathrm{DAT})\end{array}$ & 23.3 & 42.8 & 62.8 & 22.5 & 48.5 & 37.4 & 48217.50 & 241140.00 & 482.2 & $1: 5.0$ \\
\hline $\begin{array}{l}\text { Pendimethalin (pre tranplant) @ } 0.56 \mathrm{~kg} / \mathrm{ha} \\
+ \text { paraquat } 1.0 \mathrm{~kg} / \mathrm{ha} \text { (directed spray at } 45 \\
\text { DAT) }\end{array}$ & 26.1 & 46.2 & 64.2 & 22.3 & 50.0 & 37.9 & 44068.50 & 235520.00 & 471.0 & $1: 5.2$ \\
\hline Oxyfluorfen (pre tranplant) @ 0.15 kg/ha & 20.6 & 39.5 & 59.5 & 21.3 & 49.1 & 35.1 & 42184.50 & 213290.00 & 426.5 & $1: 5.0$ \\
\hline Oxyfluorfen (pre tranplant) @ $0.20 \mathrm{~kg} / \mathrm{ha}$ & 25.6 & 41.3 & 61.3 & 20.6 & 48.5 & 35.9 & 42214.50 & 217465.00 & 434.9 & $1: 5.2$ \\
\hline $\begin{array}{l}\text { Oxyfluorfen (pre tranplant) @ } 0.15 \mathrm{~kg} / \mathrm{ha}+ \\
\text { hoeing (45 DAT) }\end{array}$ & 24.6 & 44.1 & 64.1 & 23.0 & 47.1 & 39.1 & 47184.50 & 247610.00 & 489.2 & $1: 5.3$ \\
\hline $\begin{array}{l}\text { Oxyfluorfen (pre tranplant) @ } 0.15 \mathrm{~kg} / \mathrm{ha}+ \\
\text { paraquat } 1.0 \mathrm{~kg} / \mathrm{ha} \text { (directed spray at } 45 \\
\text { DAT) }\end{array}$ & 23.0 & 42.7 & 62.7 & 22.6 & 48.1 & 36.8 & 43475.50 & 215450.00 & 430.9 & $1: 4.9$ \\
\hline Trifluralin (pre tranplant) @ $60 \mathrm{~kg} / \mathrm{ha}$ & 22.1 & 43.1 & 61.1 & 19.6 & 48.5 & 35.2 & 42529.50 & 204965.00 & 409.9 & $1: 4.7$ \\
\hline Trifluralin (pre tranplant) @ 0.90 kg/ha & 20.5 & 40.7 & 60.7 & 21.0 & 47.0 & 36.2 & 42748.50 & 212130.00 & 424.2 & $1: 4.9$ \\
\hline $\begin{array}{l}\text { Trifluralin (pre tranplant) @ } 0.60 \mathrm{~kg} / \mathrm{ha}+ \\
\text { hoeing (45 DAT) }\end{array}$ & 25.0 & 43.4 & 63.2 & 23.0 & 47.5 & 38.1 & 47529.50 & 236115.00 & 477.2 & $1: 4.9$ \\
\hline $\begin{array}{l}\text { Trifluralin (pre tranplant) @ } 0.60 \\
\mathrm{~kg} / \mathrm{ha} \text { +paraquat } 1.0 \mathrm{~kg} / \mathrm{ha} \text { (directed spray at } \\
\text { 45 DAT) }\end{array}$ & 23.1 & 40.6 & 60.6 & 22.0 & 48.0 & 38.4 & 43945.50 & 234090.00 & 472.1 & $1: 5.2$ \\
\hline Black mulch & 26.0 & 44.2 & 66.4 & 25.6 & 55.5 & 45.9 & 49842.50 & 255275.00 & 510.5 & $1: 5.1$ \\
\hline Weed free & 23.1 & 41.5 & 61.8 & 25.1 & 53.4 & 43.0 & 79342.50 & 253425.00 & 506.8 & $1: 3.9$ \\
\hline Un-weeded & 19.6 & 38.8 & 58.8 & 18.0 & 37.0 & 28.7 & 41842.50 & 170530.00 & 341.0 & $1: 4.0$ \\
\hline $\mathrm{CD}(\mathrm{P}=0.05)$ & N.S & 3.6 & 2.6 & 1.7 & 6.6 & 2.7 & & & 4.2 & \\
\hline
\end{tabular}


The total weed population differed significantly among various weed control treatments. Significantly lower total weed population was noticed in weed free check.Mulching with black polyethylene resulted in better weed control efficiency as soil was covered thoroughly so there was no space for weeds to grow. Manual weeding at 45 DAT showed better result followed by treatment comprising with post-emergence application of paraquat at 45 DAT, thus showing better result than other treatments. These findings are in line with similar results obtained by Nadagouda (1995). Among the herbicides oxyfluorfen was very effective in controlling the weeds and resulted in significantly higher weed control efficiency. In present study it was noticed that plants gained maximum height of 44.2 and $66.4 \mathrm{~cm}$ after 60 and 90 DAT and number of branches recorded were 25.6. It was most probably due to the fact that in these plants due to lesser competition with weeds it encouraged growth of plant which was depicted in terms of better height and more number of branches. With respect to yield parameters, significantly highest number of fruits per plant (45.9) and average weight of fruit $(55.5 \mathrm{~g})$ were recorded with black polyethylene mulch. There was significant difference in yield of plots influenced by different treatments. It was observed that maximum yield of $510.5 \mathrm{q} / \mathrm{ha}$ was obtained from plots which were influenced by black mulch which is significantly higher than other treated plots and was at par with weed free plots also. Among herbicides maximum yield of 489.2 $\mathrm{q} / \mathrm{ha}$ was obtained in oxyfluorfen (preemergence) @ $0.15 \mathrm{~kg} / \mathrm{ha}$ + hoeing (45 DAT) which was significantly higher than other chemically treated plots. The higher yield may be attributed to lower dry matter accumulation by weeds and decrease in their population that helped increasing the yield attributes which ultimately led to higher yield (Mekki et al., 2010). In transplanted crop black mulch $\left(\mathrm{T}_{13}\right)$ recorded significantly lower total weed population as compare to other treatment plots. Among other treatments oxyfluorfen (pre-emergence) @ $0.15 \mathrm{~kg}$ a.i /ha + hoeing (45 DAT) $\left(\mathrm{T}_{7}\right)$ recorded significantly lower weed count. It is important to study economics of the experiment as no technology can be recommended without knowing its profit and loss. Maximum profit was achieved in oxyfluorfen (pre-emergence) @ $0.15 \mathrm{~kg}$ a.i $/ \mathrm{ha}+$ hoeing (45 DAT) $\left(\mathrm{T}_{7}\right)(1:$ 5.3) followed by trifluralin (pre-plant incorporation)@0.90kg a.i /ha+ gramaxone $1.0 \mathrm{~kg}$ a.i /ha (directed spray at $45 \mathrm{DAT})\left(\mathrm{T}_{12}\right)$ (1: 5.2). Black mulch showed cost: benefit of 1: 5.1 (Tables 2 and 3).

The present study is of the opinion that black polyethene mulch improves the yield and its parametres as a result of which yield was improved where it recorded a yield potential of $510.55 \mathrm{q} / \mathrm{ha}$ as compared to $341.06 \mathrm{q} / \mathrm{ha}$ in unweeded check. Among chemical treatments maximum yield was obtained with oxyfluorfen (pre-emergence) @ $0.15 \mathrm{~kg}$ a.i /ha + hoeing (45 DAT) $\left(\mathrm{T}_{7}\right)$ which recorded a yield potential of $489.22 \mathrm{q} / \mathrm{ha}$. It is thus concluded from the present investigation that oxyfluorfen (pre-emergence) @ $0.15 \mathrm{~kg}$ a.i /ha + hoeing (45 DAT) is beneficial for weed management in brinjal and also it improves the cost-benefit ratio. Oxyflourfen @ $0.15 \mathrm{~kg}$ a.i /hat hoeing (45 DAT) did not show any phyto-toxicty on brinjal plants. Hence this treatment was recorded better than rest of the treatments. It is also conclude that Trifluralin (pre-plant incorporation) @ $0.240 \mathrm{~kg} \mathrm{a.i} / \mathrm{ha}$ can be used in the nursery of brinjal.

\section{References}

Bhaduri P N (1951) Interrelationship on non tuberiferous species of Solanum with some consideration on the origin of brinjal (Solanum melongena L.). The Indian J Genet Pl Breed 11: 75-82. 
Kumar B V and Pratap M (1993) Effects of weeding at different stages on nutrienst uptake by weeds in Brinjal. $J$ Maharashtra Agric Uni 18: 426-27.

Mani V S, Gautam K C and Chakrabority P (1968) Losses in crop yield in India due to weed growth. PANS (C) 142: 141158

Mekki B B, Faida A A and Kowthar G (2010) Effect of weed control treatments on yield and seed quality of some Canola cultivars and associated weeds in newly reclaimed sandy soils. AmericanEurasian J. Agri. Env. Sci.7 (2): 202-09. management in drill sown onion (Allium cepa L.). M.Sc. (Agri.) Thesis, Univ. Agric. Sci., Dharwad, Karnataka (India) Singh N and Kalda T S (2001) Brinjal, Textbook of Vegetables, Tuber Crops and Spices. pp. 29-48 (Eds. Thamburaj $\mathrm{S}$ and Singh N). ICAR, New Delhi.

Vavilov N I (1931). The role of central Asia in the origin of cultivated plants. Bull Appl Bot-Genet Pl. Breed 26: 3-44

Walia U S (2009) Weed Management. Kalyani Publishers, New Delhi. Pp. 116.

Nadagouda B T (1995) Integrated weed

\section{How to cite this article:}

Sumeet Singh, Kulbir Singh, D.S. Khurana and Sardana, V. 2017. Economic Analysis of Integrated Weed Management in Brinjal Crop (Solanum melongena L.). Int.J.Curr.Microbiol.App.Sci. 6(12): 1245-1251. doi: https://doi.org/10.20546/ijcmas.2017.612.140 